\title{
EPR, time, irreversibility and causality
}

\author{
Umberto Lucia $^{1, a}$ \& Giulia Grisolia ${ }^{1, b}$ \\ ${ }^{1}$ Dipartimento Energia "Galileo Ferraris", Politecnico di Torino \\ Corso Duca degli Abruzzi 24, 10129 Torino, Italy \\ ${ }^{a}$ umberto.lucia@polito.it \\ ${ }^{b}$ giulia.grisolia@polito.it
}

\begin{abstract}
Causality is the relationship between causes and effects. Following Relativity, any cause of an event must always be in the past light cone of the event itself, but causes and effect must always be related to some interactions. In this paper, causality is developed as a consequence of the analysis of the Einstein, Podolsky and Rosen paradox. Causality is interpreted as the result of the time generation due to irreversible interactions of real systems among them. Time results as a consequence of irreversibility, so any state function of a system in its space cone, when affected by an interaction with an observer, moves into a light cone or within it, with the consequence that any cause must precede its effect in a common light cone.

Keyword: EPR paradox; Irreversibility; Quantum Physics; Quantum thermodynamics; Time.

The problem of the link between entropy and time has a long story and many viewpoint. In relation to irreversibility, Franklin [1] analysed some processes, in steady states, evaluating their entropy variation, which results only related

The physical science which develops the study of the energy transformations is thermodynamics, which allows the scientists to obtain fundamental results [2-4] in physics, chemistry, biophysics, engineering and information theory. During the development of this science, entropy has generally been recognized as one of the most important thermodynamic quantities for its interdisciplinary applications [5-8].
\end{abstract} to energy transformations. 
An entropy variation is always related to the evolution of the state of any system, between its initial and final states [6, 7]. There are many different approaches to describe the irreversibility in a process [2-4], and the entropy can be considered as a quantification of the irreversibility $[6,7,9,10]$. Classical thermodynamics is developed by using a small number of state variables [2-4], while, in non-equilibrium thermodynamics, the system is described by considering its subsystems [11, 12], under the assumption that each of them is in local equilibrium $[5,11,12]$. This last hypothesis is required in order to introduce temperature as a measurable quantity $[5,11,12]$. So, $T \sigma=\sum_{i} J_{i} X_{i}>0$ represents a measure of the dissipation, being $T$ the absolute thermodynamic temperature, $\sigma$ the entropy production density, i.e. the time rate of the entropy density, generated by an irreversible process, $J_{i}$ the heat and mass flows, and $X_{i}$ the generalized driving forces for vector transport processes or for chemical reactions [13-15]. Moreover, recently, a growing interest has been developed in the analysis of the fundamental role of fluxes in thermodynamics and natural systems [16-24].

Last, a scientific interest is growing in establishing a comprehensive approach to irreversible processes, based on microscopic analysis $[5-7,11,12$, 25-28].

In this context, a possible link has been proposed between the macroscopic approach to irreversibility and microscopic behaviour of a system [29], suggesting the consumption of free energy as the principle cause of far from equilibrium states. Consequently, the related entropy production is generated by the redistribution of energy, momentum, mass and charge [30].

The effort in developing a thermodynamic approach for real systems leaded to a growing interest towards the concepts of potentials and availability, in order to take into account of time in the development of the theoretical models [31].

Honig [32] developed the relation between time and entropy in irreversible processes, by considering the heat transfer through the border of the systems. His approach considers time $t$ only as a parameter to represent the path that the system follows from the initial to the final configurations.

The concept of path in a state-space leads to consider a set of consequent and related events, one followed by another caused by the previous one. But, this concept is the thermodynamic expression of causality. Indeed, causality is the relationship between causes and effects [33]. In physics, causality represents the flow of events such that the causes of an event must always be in the past light cone of the event itself and always related to some interactions of the system. In special and general relativity, the light cone is the path that a photon of light, emitted by a single source, takes through spacetime $[34,35]$. In special and general relativity, an effect cannot occur from a cause 
that is not in the past light cone of that event. Moreover, a cause cannot have an effect outside its future light cone [34, 35]. Consequently, an event cannot produce any effect if it is outside of the future light cone of another event [36].

In the second law of thermodynamics, an arrow of time is defined. But, also, causality, just because the connection between a cause and an effect, requests a direction of time [37]: this property is a characteristic just only of this physical theory. The connection between causality and entropy has recently been pointed out [37], with the consequence of defining time as the metric of causality. Moreover, time has been highlighted to be discrete in nature [37].

In summary, it seems plausible to study how to link together the concepts of time, irreversibility, and causality. The aim of this paper is to suggest a possible approach to link together the concepts of time, irreversibility, and causality, by starting from some considerations on Einstein, Podolsky and Rosen (EPR) paradox.

\section{Materials and Methods}

Since 1927, Bohr developed the principle of complementarity, a fundamental theory of quantum mechanics, based on observation and measurement [38]. The principle points out that, considering two quantum systems $\mathrm{A}$ and $\mathrm{B}$, the measurement on one of them, for example A (or B) involves a physical interaction with the experimental setup, that affects both systems. This phenomenon is uncontrollable, even if it can be predicted statistically.

Since 1935, Einstein, Podolsky and Rosen developed some criticisms on the Bohr results. The EPR paradox presents some consequences on the foundation of quantum mechanics, even if many other interesting problems were originated by this criticism. In particular the problem of the collapse of the wave function, which represents a current open problem.

In 1935 [39], Schrödinger introduced the definition of entangled states, as the quantum pure states, $|\Phi\rangle$, from an ensamble of systems, that cannot be represented by tensorial products of eigenstates of the states themselves, which analytically results:

$$
|\Phi\rangle \neq\left|\phi_{1}\right\rangle \otimes\left|\phi_{2}\right\rangle \otimes \cdots \otimes\left|\phi_{n}\right\rangle
$$

where $\otimes$ is the tensorial product, and $|\psi\rangle$ are the states in the Hilbert space $\mathcal{H}$. A state is entangled if and only if it cannot be factorized. 
During a measurement, a full wave function $|\Phi\rangle$ collapses into an eigenstate, $\left|\phi_{i}\right\rangle$, of the state bases, such that [40]:

$$
\begin{aligned}
& \left\langle\Psi|| \psi_{i}\right\rangle=1 \\
& \left\langle\Psi|| \psi_{j}\right\rangle=0, \forall j \neq i
\end{aligned}
$$

These relations express analytically the effect of the interaction between the system and the experimental set up.

Recently, the definition of time $[29,41]$ has been introduced by considering an analysis of photon-atomic-electron interaction, in relation to the irreversibility [42-44] based on an engineering thermodynamic viewpoint [4548]. Time is conjectured to be related both to the entropy production and to the entropy production rate: this result agrees to the approach of Planck and Einstein, who have pointed out that the law of evolution of a system is precisely the law of evolution of entropy [49, 50]. The problem of linking macroscopic to microscopic approaches is of interest in the analysis of various problems; indeed, it has been pointed out that macroscopic and microscopic approaches are two complementary tools [51], for studying the complex problems, where both the approaches coexist [5-7], as, for example, the relations between quantum mechanics and classical physics, matter-radiation interaction, electrodynamics of the Wheeler-Feynman model, nanothermodynamics, etc. At atomic level, photons can be absorbed by the atomic or molecule electrons, and an electronic energy transition occurs between the energy levels of two atomic stationary states. Then, the photons can be also emitted by the excited electrons when they jump down into the energy level of the original stationary state. During this phenomenon, the electrons seem to follow a reversible energetic path, because they come back to the original stationary state of low energy level [52-56]. Indeed, when we consider a single atom or molecule, the energy perturbation of the center of mass is of the order of $10^{-13} \mathrm{~J}$, while a usual energy for the electron transition, between two atomic or molecule levels, is of the order of $10^{-8} \mathrm{~J}$, with an excited state lifetime of the order of $10^{-8} \mathrm{~s}$ [55]; consequently, the approximation, of not considering the effect of the atomic nucleus, is introduced, and we need to underline that it is only an approximation [52-56], that cannot be considered in an analysis of the irreversibility which requires some considerations just on the role of the nucleus during the photon-atomic electron interaction [45$47,57]$. As a consequence of the interaction between the atomic or molecule electron and the photon, a footprint occurs in the atom or molecule. The results obtained in Refs. $[29,45,46]$ point out that the interaction between a photon and an electron in an atom affects the energy level both of the electron and of the center of mass of the atom in accordance with the theoretical and experimental results summarised in Refs. [42-44, 56, 58]. So, 
the macroscopic irreversibility is the result of the microscopic irreversibility due to the photon-electron interaction, which is the interaction between the environmental electromagnetic waves and the matter. Following the results obtained in the thermodynamic analysis of electromagnetic fields [59], this interaction can be expressed in term of the entropy production and of the entropy production rate. But, the ratio between the entropy production and of the entropy production rate are a time. In analogy with analytical mechanics, where position and velocity can be used as independent variables for the state space, we introduce entropy the entropy production $\sigma$ and the entropy production rate $\Sigma$ as the independent variables of the state space $\Omega=\{(\sigma, \Sigma)\}$, used to study the behaviour of the photon-atomic electron interaction. So, we can introduce the definition of time in relation as follows: $[41,60]$, holds to the definition of time interval as $[41,60]$ :

$$
\tau=\frac{\sigma}{\Sigma}
$$

Now, the entropy production rate can be written in relation to the electromagnetic waves as follows [59]:

$$
T_{0} \Sigma=\frac{A}{2} \varepsilon_{0} c E_{e l}^{2}+\frac{A}{2 \mu_{0}} c B_{m}^{2},
$$

where $E_{e l}$ is the electric field, $B_{m}$ is the magnetic field, $c$ is the velocity of light, a universal constant in the Universe, $\varepsilon_{0}$ is the electric permittivity in vacuum and $\mu_{0}$ is magnetic permeability in vacuum, $A$ is the area of the border of the thermodynamic control volume, and $T_{0}$ is the environmental temperature. The entropy production can be related to the analysis of irreversibility [42-44] of the interaction between a photon and an atomic electron $[41,60]$. Here, the fundamental results are summarised in order to be used in the thermodynamic analysis of EPR paradox. To do so, we consider a photon which incomes to the atomic electron. For simplicity, we consider a Hydrogen-like atom, which is an open system from a thermodynamic viewpoint. The incoming photon has an energy $E_{\gamma}=h \nu$ and a momentum $\mathbf{p}_{\gamma}=h \nu \mathbf{u}_{c} / c$, where $h=6.62607004 \times 10^{-34} \mathrm{~J} \mathrm{~s}$ is the Planck constant, $\nu$ is the frequency of the electromagnetic wave, mathbf $u_{c}$ is the versor of the speed of light, and $c=299792458 \mathrm{~m} \mathrm{~s}^{-1}$ is the speed of light [61]. If the photon has a frequency $\nu=\left(E_{f}-E_{i}\right) / h$, being $E_{i}$ the energy of the ground state of the electron, $E_{f}$ the energy of the excited level, the electron absorbs the photon and jumps from the ground state into an excited energy state. After the lifetime of this state, on which some considerations have previously been introduced, the electron jumps down into the fundamental state, emitting a new photon. there exists a change in the kinetic energy of the 
center of mass of the atom, but its amount $\left(10^{-13} \mathrm{~J}\right)$ is usually negligible in relation to the energy change $\left(10^{-8} \mathrm{~J}\right)$ in electronic transition and its time of occurrence $\left(10^{-13} \mathrm{~s}\right)$ is greater than the time of electronic transition $\left(10^{-15}\right.$ s) $[45,55,62]$. But, if we develop a thermodynamic analysis of irreversibility, we must take into account this effect, so that the final energy of the atom, after the photon has been absorbed, results [45, 55, 62]:

$$
E_{f}=E_{i}+h \nu-\frac{h^{2} \nu^{2}}{2 M c^{2}}
$$

where $M$ is the mass of the atom, and, in an analogous way, when the photon is emitted, it follows $[45,55,62]$ :

$$
E_{i}=E_{f}-h \nu-\frac{h^{2} \nu^{2}}{2 M c^{2}}
$$

Consequently, we expect a footprint in the atom $[45,46,62]$ because, if we don't use the approximation of neglecting the effect on the center of mass, the interaction, between a photon and an electron in an atom, affects both the energy level of the electron and the energy level of the center of mass of the atom. Here, we stress that this effect is well known in quantum physics, even if the approximation of neglecting it is usually accepted, because of the small energy contribution of the center of mass. The quantum state function, after this interaction, solution of the Schrödinger equation, can be obtained by the usual quantum mechanical approach [61, 63]. So, it was analytically shown that the macroscopic irreversibility is the consequence of the microscopic irreversibility due to the interaction photon-electron, or from a macroscopic point of view, between the electromagnetic waves and the matter. The fundamental state function, before the interaction, solution of the Schrödinger equation can be obtained by the quantum mechanics $[61,63]$ :

$$
\psi(\mathbf{r}, \mathbf{R})=\phi(\mathbf{r}) \vartheta(\mathbf{R})
$$

where $\phi$ is the wave function of the electron, $\mathbf{r}=\mathbf{r}_{N}-\mathbf{r}_{e}$ are the relative coordinates, with $\mathbf{r}_{N}$ the coordinates of the atomic nucleus and $\mathbf{r}_{e}$ the coordinates of the atomic electron, and $[45,46,62]$ :

$$
\begin{aligned}
\vartheta(\mathbf{R}) & =\frac{1}{(2 \pi)^{3 / 2}} \exp (i \mathbf{k} \cdot \mathbf{R}) \\
\mathbf{k} & =\sqrt{\frac{2 M}{\hbar} E_{C M}} \mathbf{u}_{C M}
\end{aligned}
$$

where $\mathbf{u}_{C M}$ is the versor of the nucleus momentum, $E_{C M}=\mathbf{P}^{2} / 2 M$ is the kinetic energy of the center of mass, $\mathbf{R}=\left(m_{N} \mathbf{r}_{N}+m_{e} \mathbf{r}_{e}\right) /\left(m_{N}+m_{e}\right)$ is the 
coordinates of the center of mass before photon-atomic electron interaction, $M$ is the total mass of the atom, $m_{e}$ is the mass of the electron and $m_{N}$ is the mass of the nucleus, $\mathbf{P}$ is the momentum of the center of mass, $\hbar=h / 2 \pi$ where $h$ is the Palnck constant, and $\mathbf{u}_{C M}$ is the versor of the momentum of the nucleus. Then, the photon incomes to the atomic electron which jumps from the fundamental state into an excited energy state and then it jumps down to the fundamental state, with the emission of a new photon. The fundamental state function, after this interaction, solution of the Schrödinger equation can be obtained by the quantum mechanics $[29,45,46,55,61,63]$ :

$$
\psi_{f}(\mathbf{r}, \mathbf{R})=\phi(\mathbf{r}) \vartheta_{f}(\mathbf{R})
$$

with $\phi$ wave function of the electron and $[45,46,55,62]$ :

$$
\begin{aligned}
\vartheta_{f}(\mathbf{R}) & =\frac{1}{(2 \pi)^{3 / 2}} \exp \left(i \mathbf{k}^{\prime} \cdot \mathbf{R}\right) \\
\mathbf{k}^{\prime} & =\sqrt{\frac{2 M}{\hbar}\left(E_{C M}+\frac{m_{e}}{M} E_{p h}\right)}\left(\mathbf{u}_{C M}+\mathbf{u}_{c}\right)
\end{aligned}
$$

where $E_{p h}$ is the energy of the incoming photon, and $\mathbf{u}_{c}=\mathbf{c} / c$ is the versor of propagation of the electromagnetic wave, with $\mathbf{c}$ the velocity of light and $c$ its value. A quantum thermodynamic approach to this photon-atomic electron interaction, allows us to prove that this atomic process leaves the footprint $[29,45,46]$ :

$$
E_{f t p}=\Delta E_{p h}=\Delta E_{C M}=\left\langle\psi(\mathbf{r}, \mathbf{R})|\mathcal{H}| \psi_{f}(\mathbf{r}, \mathbf{R})\right\rangle=\frac{m_{e}}{M} E_{p h}
$$

where $\mathcal{H}$ is the Hamiltonian of the photon-atomic electron interaction, i.e. from a macroscopic point of view, the interaction between electromagnetic wave and matter. Then, it is possible to evaluate the entropy production as follows $[29,45,46]$ :

$$
T_{0} \sigma=\frac{m_{e}}{M} E_{p h}
$$

Some considerations may be introduced [64]:

- Time is the result of the irreversibility;

- Locally, entropy can decrease, but the entropy production (due to irreversibility) must always increase, with the consequence that time can only increase. 


\section{Results}

In relation to the EPR paradox, our result can be summarised stating that without any interaction, time cannot exist, as previously suggested as a consequence of the interaction between electromagnetic waves and matter. Following the original EPR gedanken-experiment [65], we consider two spin-1/2 particles, generated by the same source, A and B, which move in opposite directions. These particles can be detected only if they interact with an experimental setup. Until they interact, from the previous considerations, they are only in spatial dimensions, because time flow starts only from their interaction with an observer. So, independently by their distance, the two systems are not aware of being separate, because they aren't subjected to time dimension. Before the interaction with the observer, their state function is $[66]$ :

$$
|\Phi\rangle=\frac{1}{\sqrt{2}}\left(\left|\frac{1}{2}\right\rangle_{\mathrm{A}}\left|-\frac{1}{2}\right\rangle_{\mathrm{B}}-\left|-\frac{1}{2}\right\rangle_{\mathrm{A}}\left|\frac{1}{2}\right\rangle_{\mathrm{B}}\right)
$$

So, as a consequence of the interaction of one of the particle with the set up, the state function collapses into a particular value, determined by the interaction, and, as a consequence of the interaction, also time begins to flow. So, during the collapse, the particles can influence one another, because still outside of time, up to the end of the interaction, so, for example

$$
|\phi\rangle_{A}=\left|\frac{1}{2}\right\rangle \Rightarrow|\phi\rangle_{B}=\left|-\frac{1}{2}\right\rangle
$$

The results obtained confirm the Bohr approach, but highlight also the fundamental role of the space-time, obtained by Einstein, and the recent relation between time, causality and space-time [67]. Indeed, the proof suggested points out that the conditioning of the measurements is due to the zero value of the time dimension unless one of the system interacts with the experimental setup. Consequently, it follows how irreversibility represents a constraint in the interactions, conditioning the behaviour of correlated systems.

So, causality, as seen by an observer, is related to the time generation due to irreversible interactions of real systems among them. If the events are correlated at the start, independently from their location, they maintain the correlation, while if they aren't correlated, they could be correlated only after an interaction. In particular, Equation (3) points out that without interaction time doesn't exist, so, it would be impossible to observe an order in the events. But, after any interaction, time occurs and the order of the events can be pointed out. Consequently, also the relations between two events can 
be highlighted, with particular regards to their causal relations. Moreover, in the theory of special relativity causality is related to simultaneous observerdependent [68]. Consequently, following special relativity, the cause must precede its effect in accordance to all inertial observers; moreover, in general relativity, the effect must belong to the future light cone of its cause, even if the spacetime is curved [34]. These statements mean that the cause and its effect are separated by a timelike interval, and the effect belongs to the future of its cause [34]. Here, we conjecture that time interval is originated only during the interaction between a system and an observer, so a timelike interval occurs only after this interaction. So, before this interaction, all the systems are in a 'contemporary' state.

\section{Discussion and Conclusions}

In Newtonian physics [69], an effect cannot occur before its cause [70]. In Special and General Relativity this statement has been improved by stressing that an effect cannot occur from a cause that is not in the back light cone of that event [71]. These results are the consequence of finite speed of light and of the fact that the speed of light is the maximum velocity in our Universe, so that no information can be transferred at a velocity higher than the one of the light.

Moreover, the concept of causality has deeply been improved by relating it to the meaning of the simultaneous observer-dependent [72], which states that the cause must always precede its effect in accordance with all inertial observers. Consequently, the cause and its effect are separated by a time-like interval in the space-time $[71,72]$ and a signal could be changed between these two related events at less than the speed of light.

Then, in quantum field theory, causality is closely related to the principle of locality, which is still under study because it depends on the interpretation of quantum mechanics with particular regards to quantum entanglement and Bell's Theorem [73]. Recently, in causal dynamical triangulation [67], causality has been related to the foundation of the space-time geometry [74].

All these viewpoints can be summarised by the results here obtained. Indeed, in relation to the link between the thermodynamic statistical analysis of the irreversible paths and their stochastic order [75], the energy flow, between system and environment, has been shown to select and shape the paths $[17,19-24,76-79]$. As a consequence of this interaction, irreversibility occurs also at atomic dimension. But, just this result allows us to approach the problem of causality, by starting from the analysis of the EPR paradox. In this way, time, as a sequence of ordered events, is the consequence of the 
causality, as the result of irreversibility, because of the interaction between the atomic electrons and the electromagnetic waves in the environment, due to the non-equilibrium state of our Universe [30, 47, 57, 60, 64, 80, 81].

\section{References}

[1] W. S. Franklin, On entropy, Physical Review 30, 766-775 (1910).

[2] J. A. Beattie and I. Oppenheim, Principles of Thermodynamics, Elsevier, Amsterdam, 1979.

[3] E. A. Guggenheim, Thermodynamics. An Advanced Treatment for Chemists and Physicists, Elsevier, Amsterdam, 1985.

[4] P. T. Landsberg, Thermodynamics and Statistical Mechanics, Dover Publications, New York, 1990.

[5] A. L. Kuzemsky, Statistical Mechanics and the Physics of Many-Particle Model Systems, World Scientific, Singapore, 2017.

[6] A. L. Kuzemsky, Temporal evolution, directionality of time and irreversibility, Rivista del Nuovo Cimento 41, 513-574 (2018).

[7] A. L. Kuzemsky, In search of time lost: Asymmetry of time and irreversibility in natural processes, Foundations of Science 25 (2020).

[8] S. K. Lin, Diversity and entropy, Entropy 1, 1-3 (1999).

[9] R. M. Velasco, L. S. García-Colín, and F. J. Uribe, Entropy production: Its role in non-equilibrium thermodynamics, Entropy 13, 82-116 (2011).

[10] G. A. Casas, F. D. Nobre, and E. M. F. Curado, Generalized entropy production phenomena: A master-equation approach, Physical Review E 89, 012114 (2014).

[11] D. N. Zubarev, Nonequilibrium Statistical Thermodynamics, Consultant Bureau, New York, 1974.

[12] A. L. Kuzemsky, Theory of transport processes and the method of the nonequilibrium statistical operator, International Journal of Modern Physics 21, 2821-2949 (2007).

[13] S. R. de Groot and P. Mazur, Non-Equilibrium Thermodynamics, NorthHolland, Amsterdam, 1962. 
[14] P. Glansdorff and I. Prigogine, Thermodynamic Theory of Structure, Stability and Fluctuations, Wiley, New York, 1971.

[15] R. Haase, Thermodynamics of Irreversible Processes, Addison-Wesley, Reading, 1969.

[16] A. Bejan, Why the Days Seem Shorter as We Get Older, European Review 27, 187-194 (2019).

[17] A. Bejan, Why the bigger live longer and travel farther: Animals, vehicles, rivers and the winds, Scientific Reports 2, 594 (2012).

[18] G. Lorenzini and C. Biserni, The constructal law: From design in nature to social dynamics and wealth as physics, Physics of Life Reviews 8 , 259-260 (2011).

[19] A. Bejan, Shape and Structure, from Engineering to Nature, Cambridge University Press, Cambridge, 2000.

[20] A. Bejan and S. Lorente, Design with Constructal Theory, John Wiley \& Sons, Hoboken, 2008.

[21] A. Bejan and S. Lorente, The constructal law and the evolution of design in nature, Philosophical Transaction B 1545, 365 (2010).

[22] A. H. Reis, Constructal theory: from engineering to physics, and how flow systems develop shape and structure, Applied Mechanics Review 59, 262-282 (2006).

[23] A. F. Miguel, The physics principle of the generation of flow configuration, Physics of Life Review 8, 243-244 (2011).

[24] A. Bejan and S. Lorente, The Physics of Life: The Evolution of Everything, St. Martin's Press, New York, 2016.

[25] M. C. Mackey, The dynamic origin of increasing entropy, Reviews of Modern Physics 61, 981-1015 (1989).

[26] M. C. Mackey, Time's Arrow: The Origin of Thermodynamic Behavior, Springer, Berlin, 1992.

[27] W. G. Hoover and C. G. Hoover, Time-irreversibility is hidden within Newtonian mechanics, Molecular Physics 116(21-22), 3085-3096 (2018). 
[28] J. Lighthill, The recently recognized failure of predictability in Newtonian dynamics, Proceedings of the Royal Society 407, 35-50 (1986).

[29] U. Lucia and E. Açikkalp, Irreversible thermodynamic analysis and application for molecular heat engines, Chemical Physics 494, 47-55 (2017).

[30] U. Lucia and G. Grisolia, Nonequilibrium Temperature: An Approach from Irreversibility, Materials 14, 2004 (2021).

[31] B. Andresen, R. S. Berry, M. J. Ondrechen, and P. Salamon, Thermodynamics for processes in finite time, Accounts of Chemical Research 17, 266-271 (1984).

[32] J. Honig, On the entropy of a class of irreversible processes, Entropy 15, 2975-2988 (2013).

[33] M. Espinoza, Théorie du déterminisme causal, L'Harmattan, Paris, 2006.

[34] S. Weinberg, Gravitation and Cosmology: Principles and Applications of the General Theory of Relativity, John Wiley \& Sons, New York, 1972.

[35] R. Penrose, The Road to Reality, Vintage Books, London, 2005.

[36] D. Bohm, Causality and Chance in Modern Physics, Taylor and Francis, London, 2005.

[37] R. Riek, Entropy Derived from Causality, Entropy 22, 647 (2020).

[38] D. Howard, Who invented the Copenhagen Interpretation? A study in mythology, Philosophy of Science 71, 669-682 (2004).

[39] E. Schrödinger, Die gegenwärtige Situation in der Quantenmechanik, Naturwissenschaften 23, 807-812 (1935).

[40] D. J. Griffiths, Introduction to Quantum Mechanics, Pearson Prentice Hall, Upper Saddle River, 2005.

[41] U. Lucia and G. Grisolia, Time: A footprint of irreversibility, Atti dell'Accademia Peloritana dei Pericolanti 97, SC1-SC4 (2019).

[42] R. O. Doyle, Great Problems in Philosophy \& Physics - Solved?, Information Philosopher, Cambridge, 2016. 
[43] R. O. Doyle, The Origin of Irreversibility, Information Philosopher, www.informationphilosopher.org, 2019 (Last access).

[44] R. O. Doyle, The continuous spectrum of the hydrogen quasi-molecule, Journal of Quantitative Spectroscopy and Radiative Transfer 492, 15551569 (1968).

[45] U. Lucia, Macroscopic irreversibility and microscopic paradox: A Constructal law analysis of atoms as open systems, Scientific Reports 6, 35792 (2016).

[46] U. Lucia, Unreal perpetual motion machine, Rydberg constant and Carnot non-unitary efficiency as a consequence of the atomic irreversibility, Physica A 492, 962-968 (2018).

[47] U. Lucia, Considerations on non equilibrium thermodynamics of interactions, Physica A 447, 314-319 (2016).

[48] H. B. Callen, Thermodynamics and an Introduction to Thermostatistics, 2nd Ed., John Wiley and Sons, New York, 1985.

[49] J.-P. Badiali, The concept of entropy. Relation between action and entropy, Condensed Matter Physics 8, 655-664 (2005).

[50] A. Einstein, Autobiographical Notes (Translated by Schilpp, P.A.), Open Court Publishing Company, Chicago, 1982.

[51] R. S. Berry and B. M. Smirnov, Clusters as tools to link macro and micro approaches, Computational and Theoretical Chemistry 1021, 2$6(2013)$.

[52] J. Franck, Elementary processes of photochemical reactions, Transactions of the Faraday Society 21, 536 - 542 (1926).

[53] E. Condon, A theory of intensity distribution in band systems, Physical Review 28, 1182-1201 (1926).

[54] M. Born and J. R. Oppenheimer, Zur Quantentheorie der Molekeln, Annalen der Physik 32, 457-484 (1927).

[55] M. Alonso and E. J. Finn, Fundamental University Physics. Vol. III. Quantum and Statistical Physics, Addison Wesley, Boston, 1968.

[56] J. C. Slater, Quantum Theory of Matter, McGraw-Hill, New York, 1951. 
[57] U. Lucia, Quanta and entropy generation, Physica A 419, 115-121 (2015).

[58] E. Condon, Nuclear motions associated with electron transitions in diatomic molecules, Physical Review 32, 858 - 872 (1928).

[59] G. P. Beretta and E. P. Gyftopoulos, Electromagnetic Radiation: A Carrier of Energy and Entropy, Journal of Energy Resources Technology 137, 021005 (2015).

[60] U. Lucia and G. Grisolia, Time: a Constructal viewpoint \& its consequences, Scientific Reports 9, 10454 (2019).

[61] R. Feynman, R. B. Leighton, and M. Sands, The Feynman Lectures on Physics. Vol. III, Addison Wesley, Boston, 1964.

[62] U. Lucia, Electron-photon Interaction and Thermal Disequilibrium Irreversibility, International Journal of Quantum Foundations 3, 24-30 (2017).

[63] L. D. Landau and E. M. Lifshitz, Quantum Mechanics, Pergamon Press, Oxford, 1965.

[64] U. Lucia, G. Grisolia, and A. L. Kuzemsky, Irreversibility and Entropy Production in Nonequilibrium Systems, Entropy 22, 887 (2020).

[65] D. Bohm, Quantum Theory, Prentice Hall, New York, 1951.

[66] M. D. Reid, P. D. Drummond, W. P. Bowen, E. G. Cavalcanti, P. K. Lam, H. A. Bachor, U. L. Andersen, and G. Leuchs, Colloquium: The Einstein-Podolsky-Rosen paradox: From concepts to applications, Review of Modern Physics 81, 1727 (2009).

[67] R. Loll, J. Ambjørn, and J. Jurkiewicz, Quantum gravity: the art of building spacetime, in Approaches to Quantum Gravity. Toward a New Understanding of Space, Time and Matter, edited by D. Oriti, Cambridge University Press, Cambridge, 2009.

[68] A. Einstein, Zur Elektrodynamik bewegter Koerper, Annalen der Physik 17, 891-921 (1905).

[69] Newton, I. (translated by Cohen, I. B. \& Whitman, A.), The Principia, 3rd Edition (1726), University of California Press, Berkeley, 1999. 
[70] R. P. Feynman, The Feynman Lectures on Physics. Vol. 1 (2nd ed.), Addison Wesley, Boston, 2005.

[71] A. Einstein, Relativity: The special and general theory, Routledge Classics, London, 1920.

[72] W. Pauli, Theory of Relativity, Pergamon Press, London, 1958.

[73] M. Kafatos, Bell's Theorem, Quantum Theory and Conceptions of the Universe, Springer Netherlands, Amsterdam, 1989.

[74] D. B. Malament, Topics in the Foundations of General Relativity and Newtonian Gravitation, University of Chicago Press, Chicago, 2012.

[75] U. Lucia, Statistical approach of the irreversible entropy variation, Physica A 387, 3454-3460 (2008).

[76] U. Lucia, Entropy generation: From outside to inside!, Chemical Physics Letters 583, 209-212 (2016).

[77] A. Bejan, Advanced Engineering Thermodynamics, John Wiley, New York, 2006.

[78] A. H. Reis, Design in nature, and the laws of physics, Physics of Life Review 8, 255-256 (2011).

[79] A. H. Reis, Use and validity of principles of extremum of entropy production in the study of complex systems, Annals of Physics 346, 22-27 (2014).

[80] U. Lucia and G. Grisolia, Time \& Clocks: A thermodynamic approach, Results in Physics 16, 102977 (2020).

[81] U. Lucia, Second law considerations on the third law: From Boltzmann and Loschmidt paradox to non equilibrium temperature, Physica A 444, 121-128 (2016). 